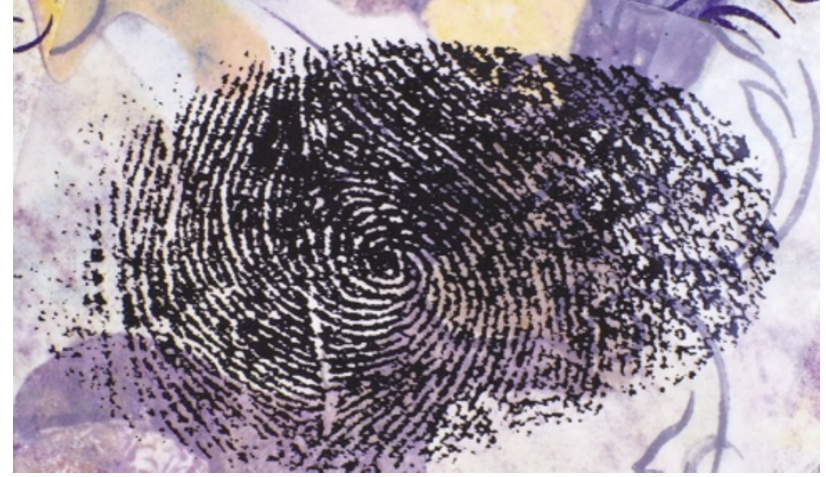

SCREENING

\section{Fingerprinting cancer cells}

Since the prostate-specific antigen (PSA) screening test was introduced into clinical practice in the early 1990s, detection of prostate cancer has greatly increased and patient management has improved. However, despite the sensitivity of this test, PSA lacks specificity as a serum marker for prostate cancer. Consequently, the need for discovery of more specific biomarkers to improve the early detection of the disease is paramount. Recent technological advances have made this possible, and, in the 1 July issue of Cancer Research, George L. Wright and co-workers describe how they have used a serum-protein fingerprinting technique to help accurately distinguish between prostate cancer, benign prostate hyperplasia (BPH) and healthy tissue.

The authors used a protein-biochip surface-enhanced laserdesorption/ionization (SELDI) mass spectrometry approach to detect proteins that were affinity-bound to a protein-chip array. They then used an artificial intelligence learning algorithm to reduce the number of proteins found down to the number that are required to differentiate prostate cancer from noncancer cohorts. Serum samples were taken from 167 patients with prostate cancer, 77 patients with $\mathrm{BPH}$ and 82 unaffected healthy men. SELDI mass spectrometry detected 779 protein peaks after clustering and peak alignment, which were narrowed down to nine protein masses necessary for classification.

So, how specific is this classification method? The overall sensitivity was $83 \%$, the specificity was $97 \%$ and the positive predictive value was $96 \%$ for differentiating prostate cancer from $\mathrm{BPH}$ and unaffected healthy men. This compares very favourably with the PSA test, in which sensitivity is $>\mathbf{9 0} \%$ but specificity is only $25 \%$. It follows that use of the serum-fingerprinting method will lead to a substantial reduction in unnecessary biopsies something which causes considerable anxiety in men who, if classified accurately, would not have needed a biopsy.

Other advantages of this screening technique include earlier detection of prostate cancer - the authors' experience suggests that prostate cancer might be detected 5 or more years earlier with this fingerprinting approach than with PSA screening. Because of tumour microheterogeneity, it is perhaps not surprising that the use of multiple biomarkers is likely to be more effective than use of a single marker. The next step is to identify other biomarkers that can differentiate aggressive cancers from nonaggressive cancers, to make this classification system for early detection as effective as possible.

Ezzie Hutchinson

(2) References and links

ORIGINAL RESEARCH PAPER Adam, B.-L. et al. Serum protein fingerprinting coupled with a pattern-matching algorithm distinguishes prostate cancer from benign prostate hyperplasia and healthy men. Cancer Res. 62, 3609-3614 (2002) WEB SITES

Encyclopedia of Life Sciences: http://www.els.net

prostate cancer

George L. Wright's lab: http://www.evms.edu/micro/index.html

\section{TRIAL WATCH}

\section{Hormone-replacement risks}

Two studies published in the 17 July issue of JAMA indicate that hormonereplacement therapy in post-menopausal women increases the risk of cancer. The Women's Health Initiative (WHI) investigators report the discontinuation of one arm of a multicentre randomized trial, which analysed the effects of combined oestrogen and progestin in healthy menopausal women. On 31 May 2002, after a mean of 5.2 years of follow-up the WHI's data- and safety-monitoring board recommended stopping the trial because results indicated that the overall health risk of the trial exceeded the benefits. The trial, scheduled to run until 2005, involved 16,608 women aged 50-79 years with an intact uterus. Women were randomly assigned to receive a daily dose of oestrogen plus progestin or placebo.

The oestrogen plus progestin group compared with placebo resulted in an increase in strokes, heart attacks, venous thromboembolism, cardiovascular disease and a $26 \%$ increase in breast cancer. However, it was also associated with a $37 \%$ decrease in colorectal cancer and a reduction in hip fractures.

The National Cancer Institute (NCI) have also reported that women who received oestrogen-replacement therapy after menopause were at increased risk for ovarian cancer. The researchers followed 44,241 women, who had been enrolled in a mammography-screening programme from 1979 to 1998. Compared with postmenopausal women, who did not receive hormonereplacement therapy, users of oestrogen-only therapy had a $60 \%$ greater risk of developing ovarian cancer, which increased with length of oestrogen use.

ORIGINAL RESEARCH PAPERS Writing Group for the Women's Health Initiative Investigators. Risks and benefits of oestrogen plus progestin in healthy postmenopausal women. JAMA 288, 321-333 (2002) Lacey, J. V. et al. Menopausal hormone replacement therapy and risk of ovarian cancer. JAMA $\mathbf{2 8 8}$, 334-341 (2002)

\section{Attack on breast cancer}

The first analysis of the ATAC (Arimidex, tamoxifen, alone or in combination) randomized trial is reported in the 22 June issue of The Lancet. It indicates that the aromatase inhibitor anastrozole (Arimidex) is an effective and well-tolerated alternative to the 'gold standard' tamoxifen as adjuvant treatment for postmenopausal women with early hormonereceptor-positive breast cancer, although longer follow-up is required before a final assessment of benefits and risks can be made.

The 9,366 patients recruited were given anastrozole, tamoxifen or a combination of the two. Anastrozole showed significantly better disease-free survival at 3 years $(89.4 \%$ versus $87.4 \%)$ and the risk of recurrence decreased by $22 \%$ compared with tamoxifen; the results with the combination therapy were similar to tamoxifen alone. The improvement in effectiveness was seen in only the $84 \%$ of patients who were hormone-receptor positive. In addition, a striking reduction in the incidence of contralateral breast cancer was seen with anastrozole compared with tamoxifen and, if this trend persists, anastrozole might prevent up to $80 \%$ of hormone-receptor-positive breast cancers.

The side-effect profiles were more complex. Anastrozole was superior to tamoxifen with respect to the incidence of endometrial cancer and thromboembolic disease, but tamoxifen was significantly better tolerated for musculoskeletal disorders and bone fractures. The difference in mechanisms of action of anastrozole and tamoxifen probably accounts for the difference in effects on the endometrium and protection of bone.

The clinicians conclude that: "...these results could be as significant to breast cancer treatment as the results first seen with tamoxifen nearly 20 years ago".

ORIGINAL RESEARCH PAPER The ATAC Trialists' Group. Anastrozole alone or in combination with tamoxifen versus tamoxifen alone for adjuvant treatment of postmenopausal women with early breast cancer: first results of the ATAC randomised trial. Lancet 359, 2131-2139 (2002) 